\title{
PARÂMETROS CLÍNICOS RELEVANTES PARA O DIAGNÓSTICO DIFERENCIAL ENTRE LEISHMANIOSE CUTÂNEO-MUCOSA E PARACOCCIDIOIDOMICOSE
}

\author{
Arnaldo Lopes Colombo, Luís Fernando Aranha Camargo, \\ Olga Gompertz Fischman e Adauto Castelo
}

\begin{abstract}
Foram estudados retrospectivamente os prontuários de 15 pacientes portadores de leishmaniose e 28 pacientes de paracoccidioidomicose, nas formas de acometimento de mucosa oronasofaringolaringeana, atendidos na Escola Paulista de Medicina de 1986 a 1990. Estes pacientes foram comparados com relação às seguintes variáveis: sexo, idade, tempo de evolução da doença, topografia da lesão e queixas clínicas. Sexo, tempo de evoluçãoda doença, topografia de lesão em septo nasal, pálatos, língua e lábios, e queixa clínica com relação a dor em cavidade oral, disfagia/odinofagia o obstrução nasal apresentaram diferença estatisticamente significante em sua ocorrência nos dois grupos de pacientes. Os autores consideram estes achados relevantes na diferenciação clínica entre as duas moléstias.
\end{abstract}

Palavras-chaves: Paracoccidiodomicose. Leishmaniose.

O Brasil é o país da América Latina que registra a maior casuística de paracoccidioidomicose, particularmente nas regiões Centro-oeste, Sul e Sudeste ${ }^{23}$. Trata-se de micose sistêmica causada pelo Paracoccidioides brasiliensis, com predileção por habitantes e/ou trabalhadores de zona rural, e que acarreta lesões tegumentares como uma de suas principais manifestações clínicas. Estes pacientes freqüentemente apresentam ulcerações em mucosa oronasofaringolaringeana 289.

Da mesma forma, a leishmaniose cutânea é endêmica em nosso país com maior ocorrência na Amazônia, região Nordeste e alguns estados do Sudeste, principalmente Minas Gerais e Espírito Santo $^{7}$. As leishmanias dos complexos brasiliensis e mexicana são responsáveis pela maioria dos casos. Esta moléstica pode causar envolvimento de mucosa oronasofaringolaringeana em $3 \%$ a $6 \%$ dos pacientes tratados para as formas cutâneas (estimando-se que tal porcentagem chegue a $20 \%$ dos casos não tratados), o que é mais comum quando o agente é $L$. b. braziliensis ${ }^{6}$, embora recentemente agentes dos complexos mexicana e donovani tenham sido implicados ${ }^{1}$. As lesões de

Trabalho da Escola Paulista de Medicina, São Paulo, SP. Enderego para correspondência: Prof. Adauto Castelo Filho, Av. Jandira 610/32, 04080-004 São Paulo, SP. Fax: (011) 549 2127, Fone: (011) 5490158.

Recebido para publicação em 11/05/92. mucosas destes pacientes envolvem lábios, pálatos, septo cartilaginoso e até laringe ${ }^{5}$.

Considerando-se a frequente sobreposição clínica e epidemiológica entre os portadores de paracoccidioidomicose e leishmaniose tegumentar, realizou-se este trabalho com o objetivo de identificar características clínicas e demográficas que contribuam para a diferenciação diagnóstica entre as duas doenças.

\section{MATERIAL E MÉTODOS}

Procedeu-se avaliação retrospectiva de todos os prontuários dos pacientes atendidos com diagnóstico de paracoccidioidomicose ou leishmaniose mucosa durante o período de janeiro de 1986 a dezembro de 1990, na Disciplina de Doenças Infeccioas e Parasitárias da Escola Paulista de Medicina.

Os critérios de inclusão neste estudo foram:

1. leishmaniose mucosa: presença de dois ou mais dos seguintes itens: clínico, epidemiológico, reação de Montenegro positiva (5 ou mais mm de induração após 72 horas), anatomopatológico compatível (com ou sem identificação do agente) e cultura da lesão.

2. paracoccidioidomicose: presença de lesão de mucosas cujo diagnóstico foi confirmado pela identificação do $P$. brasiliensis por exame microscópico direto ou cultura de material biológico da lesão.

Os pacientes portadores de leishmaniose e os de 
Colombo AL, Camargo LFA, Fischman OG, Castelo A. Parâmetros clínicos relevantes para o diagnóstico diferencial entre leishmaniose cutâneo-mucosa e paracoccidioidomicose. Revista da Sociedade Brasileira de Medicina Tropical 25:171-175, jul-set, 1992.

paracoccidioidomicose foram comparados com relação aos seguintes parâmetros: sexo, idade, queixa clínica que motivou busca de auxílio médico, tempo de evolução da doença, e topografia da lesão de mucosa.

Utilizaram-se os testes de Mann-Whitney e Fisher para comparação entre os dois grupos. As análises estatísticas foram realizadas com o pacote EPISTAT. Todos os valores de $\mathrm{P}$ foram bicaudais. As diferenças entre os dois grupos foram consideradas estatisticamente significantes para valores de $\mathrm{p}<0,05$.

A seguir, são enumerados alguns dos conceitos utilizados para definir as variáveis estudadas bem como os critérios diagnósticos:

1. Tempo de evolução da lesão: tempo entre o início dos sintomas da lesão mucosa e o diagnóstico da doença, diagnóstico este não necessariamente realizado em nosso serviço.

2. Isolamento do agente: considerado como visualização de formas amastigotas de leishmanias pela coloração de Giemsa em material de biópsia e formas típicas de $P$. braziliensis visualizadas à microscopia óptica em material de secreção de lesão mucosa com $\mathrm{KOH}$ ou em material de biópsia por coloração com prata metenamina ou hematoxilinaeosina. Não foram realizadas biópsias de todas as lesões de um mesmo paciente.

3. Topografia da lesão: definida por visualização direta de lesão mucosa sugestiva das doenças em estudo por exame otorrinolaringológico do paciente. Apesar da comprovação etiológica não ter sido repetida em todas as lesões, estas só foram consideradas manifestação da moléstia diagnosticada quando apresentaram resposta satisfatória à terapêutica específica.

4. Tratamento realizado: para leishmaniose, Glucantime $^{\mathrm{R}}$ na dose de $60 \mathrm{mg} / \mathrm{kg}$ do sal de antimônio por um período que variou de 25 a 45 dias, ou anfotericina $\mathrm{B}$ com dose total de 1,0 g. Para paracoccidioidomicose, os pacientes foram tratados inicialmente com anfotericina $\mathrm{B}$ ( 1 a 2 $\mathrm{g} /$ dose acumulada individual), sulfametoxazoltrimetoprima (1600-320 mg/dia por 6 meses) ou ketoconazol ( $400 \mathrm{mg} / \mathrm{dia}$ por 2 meses e 200 $\mathrm{mg} /$ dia por mais 4 meses). Todos os portadores desta micose receberam ainda sulfadoxina $(1 \mathrm{~g} /$ semana) ou sulfametoxazol-trimetoprima (800-
$160 \mathrm{mg} /$ diariamente) como terapêutica de manutenção, durante o período de seguimento.

\section{RESULTADOS}

Foram identificados 28 pacientes portadores de paracoccidioidomicose, com idade entre 25 e 80 anos, mediana de 50 anos, todos do sexo masculino com tempo de evolução de lesão variando de 2 a 72 meses, mediana de 6 meses. Os locais mais frequentes de lesão mucosa assim como as principais queixas clínicas acham-se resumidos na Tabela 1.

Os 15 pacientes com leishmaniose apresentaram idade variando de 30 a 72 anos, com mediana de 62 anos. Embora a maioria fosse do sexo masculino, $27 \%$ da amostra eram compostos por mulheres. $\mathrm{O}$ tempo de evolução da doença variou de 5 a 648 meses, mediana de 36 meses, sendo importante ressaltar que tal dado pode ser obtido em apenas 10 dos 15 pacientes.

O septo nasal foi o local mais comumente envolvido, com todos os pacientes apresentando lesão de septo cartilaginoso, perfurativa ou não. Em tais lesões, foi possível identificar o agente em apenas $26 \%$ dos casos. As demais lesões mucosas bem como as principais queixas clínicas dos portadores de leishmaniose acham-se resumidas na Tabela 1.

A Tabela 1 ilustra as características clínicas e demográficas estudadas nos dois grupos de pacientes, assim como a comparação entre as mesmas. Observou-se diferença estatisticamente significante no comportamento das seguintes variáveis: sexo, tempo de evolução da doença, topografia de lesão em septo nasal, pálatos, língua, e lábios, e queixas clínicas como obstrução nasal, dor em cavidade oral e disfagia/odinofagia.

\section{DISCUSSÃO}

As características demográficas apresentadas pelos pacientes com paracoccidioidomicose estudados sobrepõem-se àquelas já estabelecidas na literatura: predomínio do sexo masculino e maior prevalência de pacientes com 30 a 60 anos. $O$ tempo de evolução das lesões mucosas é compatível com o curso crônico desta moléstia granulomatosa.

Desperta interesse entretanto, pela escassez de dados publicados na literatura, a ilustração da 
Colombo AL, Camargo LFA, Fischman OG, Castelo A. Parâmetros clínicos relevantes para o diagnóstico diferencial entre leishmaniose cutâneo-mucosa e paracoccidioidomicose. Revista da Sociedade Brasileira de Medicina Tropical 25:171-175, jul-set, 1992.

Tabela 1 - Principais características das duas populações estudadas com os valores de significância estatistica da comparação entre as variáveis.

\begin{tabular}{|c|c|c|c|}
\hline Variável & $\begin{array}{l}\text { Leishmaniose } \\
\qquad(n=15)\end{array}$ & $\begin{array}{l}\text { Paracoccidioidomicose } \\
\qquad(n=28)\end{array}$ & $\mathrm{p}$ \\
\hline Sexo feminino (\%) & $27 \%$ & $0 \%$ & 0,01 \\
\hline \multicolumn{4}{|l|}{ Idade (anos) } \\
\hline Média & 55 & 50 & \\
\hline Mediana & 62 & 50 & $>0,05$ \\
\hline Variação & $30-72$ & $25-80$ & \\
\hline \multicolumn{4}{|l|}{ Tempo de evolução (meses) } \\
\hline Média & $76^{*}$ & 11 & \\
\hline Mediana & $36^{*}$ & 6 & 0,01 \\
\hline Variação & $5-684$ & $2-72$ & \\
\hline \multicolumn{4}{|l|}{ Topografia } \\
\hline Septo nasal & $100 \%$ & $0 \%$ & $<0,01$ \\
\hline Pálatos & $20 \%$ & $46 \%$ & 0,05 \\
\hline Língua & $0 \%$ & $32 \%$ & 0,01 \\
\hline Gengiva & $20 \%$ & $18 \%$ & 0,58 \\
\hline Lábios & $7 \%$ & $39 \%$ & 0,02 \\
\hline Faringe & $27 \%$ & $14 \%$ & 0,27 \\
\hline Laringe & $20 \%$ & $39 \%$ & 0,17 \\
\hline Mucosa nasal & $13 \%$ & $14 \%$ & 0,65 \\
\hline \multicolumn{4}{|l|}{ Queixa } \\
\hline Dor em cavidade oral & $7 \%$ & $57 \%$ & $<0,01$ \\
\hline Disfagia/odino & $7 \%$ & $43 \%$ & 0,01 \\
\hline Rouquidão & $13 \%$ & $39 \%$ & 0,07 \\
\hline Obstrução nasal & $100 \%$ & $0 \%$ & $<0,01$ \\
\hline
\end{tabular}

* Somente dez dos 15 pacientes foram analisados

freqüência observada em nossos pacientes dos principais sítios acometidos por lesões mucosas e suas queixas clínicas correspondentes. A mucosa de pálatos (duro ou mole) foi o sítio mais freqüentemente lesado nestes pacientes. Chama atenção ainda o envolvimento freqüente do laringe, registrado em $39 \%$ desta casuística, constituindo juntamente com os lábios, os sítios de segunda maior freqüência de acometimento de mucosas. Nenhum paciente apresentou lesão em septo nasal ou queixa clínica de obstrução nasal. As lesões mucosas eram sempre sintomáticas, levando todos os pacientes a queixarem-se de desconforto em trato digestivo superior. Da mesma forma, observou-se alta incidência de disfonia no grupo estudado (39\% dos casos). Nesta avaliação retrospectiva não foi possível detalhar o aspecto das lesões mucosas pois os prontuários não continham as informações necessárias.

Os casos de leishmaniose apresentaram padrão clínico clássico, com acometimento de septo nasal e referência de obstrução nasal como principal queixa clínica em $100 \%$ dos casos, além de baixa porcentagem de isolamento do agente da lesão. A grande maioria de casos ocorreu em homens, o que certamente tem relação com ocupação profissional, embora seja relevante o número de mulheres $(27 \%)$, refletindo provavelmente condições de moradia. $\mathrm{O}$ longo tempo decorrido (mediana de 36 meses) entre o início dos sintomas e o diagnóstico etiológico parece refletir a incapacidade diagnóstica de serviços não-especializados e também a demora do paciente em procurar assistência médica.

Diante destes dados, algumas características clínicas de utilidade no diagnóstico diferencial entreas duas moléstias foram identificadas. Enquanto $27 \%$ dos casos de leishmaniose eram mulheres, na paracoccidioidomicose todos os casos eram homens. Na totalidade dos casos de paracoccidioidomicose o envolvimento de mucosas levou a queixas 
Colombo AL, Camargo LFA, Fischman OG, Castelo A. Parâmetros clínicos relevantes para o diagnóstico diferencial entre leishmaniose cutâneo-mucosa e paracoccidioidomicose. Revista da Sociedade Brasileira de Medicina Tropical 25:171-175, jul-set, 1992.

relacionadas ao trato digestivo superior, tais como dorna lesão em cavidade oral, disfagia ou odinofagia. Em contraste, apenas um paciente comleishmaniose referiu queixas em trato digestivo superior, apesar da ocorrência de lesão mucosa em pálatos (20\%), gengiva (20\%), lábios (7\%), e faringe $(27 \%)$. Obstrução nasal crônica foi a queixa que levou todos os portadores de leishmaniose a procurar assistência médica. Provavelmente, isto contribuiu para que o tempo entre o início da doença e o diagnóstico da moléstia fosse maior nesta doença. Os pacientes com leishmaniose referiram tratamento prévio para rinite crônica durante longos periodos, sem diagnóstico etiológico. Isto pode ser atribuído à inespecificidade das queixas e à dificuldade de visualização da lesão. Já na paracoccidioidomicose, a presença de lesão oral sintomática, além de mais facilmente visualizável, denuncia manifestamente a ausência de resposta à terapêutica inespecífica. Obstrução nasal não foi referida por nenhum paciente com paracoccidioidomicose. O envolvimento do laringe, apesar de presente em ambos os grupos de pacientes, acarretou rouquidão entre os portadores de paracoccidioidomicose muito mais freqüentemente (39\%) que entre os casos de leishmaniose $(13 \%)(p=0,07)$.

Interessante observar que o diagnóstico de paracoccidioidomicose a partir de lesão mucosa é facilmente realizável através da identificação do fungo em exame microscópico direto de material coletado da mesma. Já na leishmaniose o encontro do agente na lesão mucosa é menos comum e mais laborioso. Para obter-se maior êxito no encontro deste parasita, é necessária a feitura rotineira de cultura, pesquisa direta de Leishmania da borda da lesão, e biópsia. Evidentemente, tais diferenças também contribuem para o diagnóstico mais tardio dos casos de leishmaniose.

A ocorrência de recidiva após terapêutica específica é um fenômeno conhecido nas duas moléstias, e certamente conseqüência de fatores bem distintos. Vale a pena ressaltar a alta porcentagem $(40 \%)$ de recidiva anterior ao atendimento em nosso serviço verificada para leishmaniose em comparação com os resultados obtidos para paracoccioidomicose (7\%). Esta alta taxa de recidiva observada nos pacientes com leishmaniose provavelmente foi devido à automedicação referida por vários pacientes e ao tratamento inadequado com relação à dosagem diária e acumulada de glucantime, administrado em centros não especializados.

Neste estudo, um seguimento mediano de $13 \mathrm{e}$ 10 meses dos grupos paracoccidioidomicose e leishmaniose respectivamente após tratamento específico em nosso serviço, revelou somente um caso de recidiva, sendo este um paciente com paracoccidioidomicose. Entretanto, os curtos tempos de seguimento após tratamento em nosso serviço acarretam restrições na interpretação dos baixos índices de recidiva observados.

\section{SUMMARY}

We retrospectively evaluated the medical records of 15 patients diagnosed with leishmaniasis and of 28 patientsdiagnosed with paracoccidiodomycosis presenting with involvement of the oral-nasal-pharyngeal mucosa seen at Escola Paulista de Medicina from 1986 through 1990. These patients were compared in regard to the following variables: sex, age, time since disease onset, location of lesion, and clinical complaints. Sex, time since disease onset, lesion at the nasal septum, palate, tongue, and lips, and history of oral pain, dysphagial odinophagia, and nasal obstruction were significantly different in the two groups of patients. The authors point out to the relevance of these findings in the differential clinical diagnosis of leishmaniasis and paracoccidiodomycosis.

Key-words: Paracoccidiodomycosis. Leishmaniasis.

\section{REFERÊNCIAS BIBLIOGRÁFICAS}

1. Barral A, Sampaio DP, Grimaldi Jr G, Momen H, Pratt DMM, Jesus AR, Almeida R, Badaró R, Netto MB, Carvalho EM, Jonhson Jr WD. Leishmaniasis in Bahia, Brazil: Evidence that Leishmania amazonensis produces a wide spectrum of clinical diseases. The American Journal of Tropical Medicine and Hygiene 44:536-546, 1991.

2. Franco M, Mendes RP, Bacchi MM, Iwasso MR, Montenegro MR. Paracoccidioidomycosis. Baillière's Clinical Tropical Medicine and Communicable Diseases 4:185-220, 1989.

3. Londero AT. Epidemiologia. In: Del Negro G, 
Colombo AL, Camargo LFA, Fischman OG, Castelo A. Parâmetros clínicos relevantes para o diagnóstico diferencial entre leishmaniose cutâneo-mucosa e paracoccidioidomicose. Revista da Sociedade Brasileira de Medicina Tropical 25:171-175, jul-set, 1992.

Lacaz CS, Fiorillo AM (ed) Paracoccidioidomicose, $1^{\text {a }}$. edição, EDUSP, São Paulo p.85-90, 1982.

4. Londero AT. Paracoccidioidomicose: patogenia, formas clínicas, manifestações pulmonares e diagnóstico. Jornal de Pneumologia 12:185-220, 1989.

5. Marsden PD. Mucocutaneous Leishmaniasis. British Medical Journal 301:656-657, 1990.

6. Netto EM, Marsden PD, Llanos-Cuentas EA, Costa JML, Cuba CC, Barreto AC, Badaró R, Jonhson WD, Jones TC. Long term follow-up of patients with Leishmania (viannia) braziliensis infection treated with Glucantime ${ }^{R}$. Transactions of the Royal
Society for Tropical Medicine and Hygiene 84:367370, 1990.

7. Organização Mundial de Saúde. Las Leishmanias: Informe de un comité de expertos, Genebra, 1984.

8. Paiva LJ, Nova R. Lesões orofaringolaringeanas. In: Del Negro G, Lacaz CS, Fiorillo AM (ed) Paracoccidioidomicose, $1^{\text {a. }}$ edição, EDUSP, São Paulo p.157-160, 1982.

9. Restrepo A. Paracoccidioides brasiliensis. In: Mandell GL, Douglas RG, Bennett JE (ed) Principles and practice of infectious diseases, $3^{\text {rd }}$ edition, Churchill Livingstone, New York p.2028-2031, 1990. 